\title{
Deep Ore Zone mine wet ore mining empirical learnings, mining process evolution and development pathway
}

\author{
I Edgar PT Freeport, Indonesia \\ R Prasetyo PT Freeport, Indonesia \\ M Wilkinson PT Freeport, Indonesia
}

\begin{abstract}
The Deep Ore Zone (DOZ) panel cave mine has experienced a steady increase in the prevalence of wet ore within the mine. The mine has a unique data set on this challenge and has established sufficient empirical relationships to manage the hazards associated with it. This includes an understanding of not only the probability of wet ore rushes but also the potential severity of these rushes. In parallel, the mine has progressed towards the goal of establishing a remote cave mining operation. To date, the mine has converted $100 \%$ of its Load Haul Dump (LHD) operations to remote; has updated $30 \%$ of the active chutes to remote operation; is largely complete converting its water cannons to remote operation and is in the process of finalizing the work plan to convert the mobile rock breakers and other support equipment to remote operation. All remote operations will be housed in a single location on the surface. Although driven by the safety requirements of managing wet muck, this change points to the future of mining as a continuous and remote operation.
\end{abstract}

\section{Introduction}

Wet muck is a term used at PT Freeport Indonesia (PTFI) to describe ore that has a sufficient water and fines content to present an inrush hazard. This type of ore and resultant wet muck inrush, locally termed a "spill", was first experienced in the underground operations in 1989 and is generally understood to be a consequence of comminution within the draw columns and the high rainfall experienced at the mine.

The Deep Ore Zone (DOZ) mine was established below the Intermediate Ore Zone (IOZ) mine, commencing production in 2001. The mine is a relatively large Panel Cave mine that achieved peak production of 80 ktpd in 2008 (Casten 2008) and an average of $80 \mathrm{ktpd}$ by 2010. Approaching the end of its life, the DOZ is producing $30 \mathrm{ktpd}$ today with final ore expected to be extracted in 2022. The bulk of PTFI's wet muck experience has been acquired in this mine.

During its operation, the wet muck hazard and the consequent management approach has evolved. Spills have become more frequent and very large spills, although rare, have begun to occur as the draw columns have matured. In addition, the location of inrushes has expanded from the production footprint to the haulage level, subsequently to oreflow circuit and ultimately to the mill stockpile. The mine has adapted to these changes by increasing the number of tasks completed remotely, developing spill hazard assessment criteria, increasing the pedestrian free areas and stand-off distances as well as pursuing an active blending strategy.

As a result of these changes, the mine has evolved as a prototype for an underground autonomous caving operation. This prototype has allowed PTFI to map a development plan that will see the Grasberg Block Cave (GBC) and Deep Mill Level Zone (DMLZ) mines operating autonomous, remote and manual operations seamlessly within the same Autonomous Mining Area (AMA) that is managed from a single Remote Operating Room (ROR) on the surface. 


\section{Wet muck classification and history}

The management of wet muck requires that the ore be classified in some manner to allow controls to be applied in accordance with the level of hazard. Further, a classification scheme is required to allow tracking of the spread and development of the wet muck within the production areas. The basic classification scheme at PTFI is based on the level of hazard at which remote loaders must be applied and is unchanged since its initial development.

\subsection{Wet muck classification}

Wet muck is identified by visual observation only with assessment by a small committee on a weekly basis using a simple classification scheme based on the fragmentation size and water content. The classification scheme was originally derived from a commissioned study (Call \& Nicholas 1998) and has evolved since then to that shown in Table 1. Essentially the classification seeks to identify if the material is both fine and saturated. It is subjective although some basic tests can be done in the field, for example material that will form a ball when squeezed is type 2 and material from which excess water emerges when squeezed is type 3.

\section{Table 1 Wet muck classification scheme}

\begin{tabular}{|c|c|c|c|}
\hline $\begin{array}{l}\text { Water content } \\
\text { (by mass) }\end{array}$ & $\begin{array}{l}\text { Ore } \geq 5 \mathrm{~cm}>70 \% \\
\text { (coarse material) }\end{array}$ & $\begin{array}{c}\text { Ore } \geq 5 \mathrm{~cm} 30 \%-70 \% \\
\text { (mixed material) }\end{array}$ & $\begin{array}{c}\text { Ore } \geq 5 \mathrm{~cm}<30 \% \\
\text { (fine material) }\end{array}$ \\
\hline$<8.5 \%$ (Dry) & $\mathrm{A} 1$ & B1 & C1 \\
\hline $8.5 \%-11 \%$ (Moist) & A2 & B2 & $C 2$ \\
\hline$>11 \%($ Wet $)$ & A3 & B3 & $\mathrm{C} 3$ \\
\hline
\end{tabular}

Drawpoints in green class do not pose a spill risk, yellow class drawpoints pose a low risk and can be mucked manually with constraints, red class drawpoints can only be mucked remotely. All persons working in a production area that contains wet muck must undergo training as a Wet Muck Assessor (Learning and Organisational Development 2018). This training enables each and every person in the wet muck area to determine the level of hazard of the drawpoints in their work area.

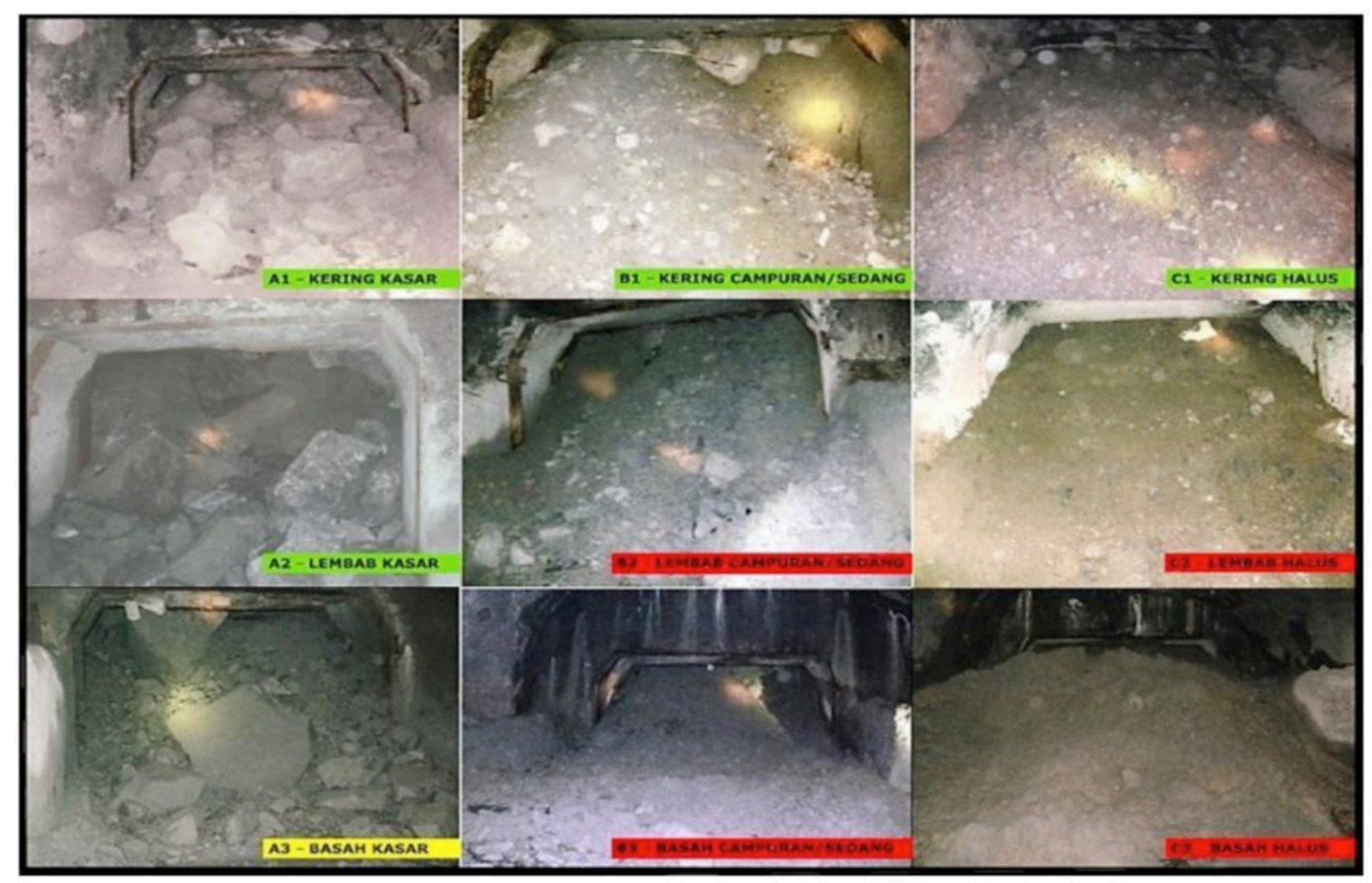

Figure 1 Examples of drawpoints showing various wet muck classes 


\subsection{Increase in active wet muck drawpoints with Time}

DOZ began experiencing wet muck in drawpoints while still constructing new drawpoints as part of the panel cave development. This provided the flexibility to produce from the new coarse and dry material in preference to the wet material. It also allowed the wet drawpoints to be closed and still maintain sufficient inventory of drawpoints to achieve the mining plan. In 2015, when the panel cave development completed, only $25 \%$ of the active drawpoints required remote mining. As can be seen in Figure 2 below, with no new dry drawpoints to replace wet drawpoints, the percentage of wet drawpoints increased rapidly and by the end of 2018 , all mining panels were being mined remotely.
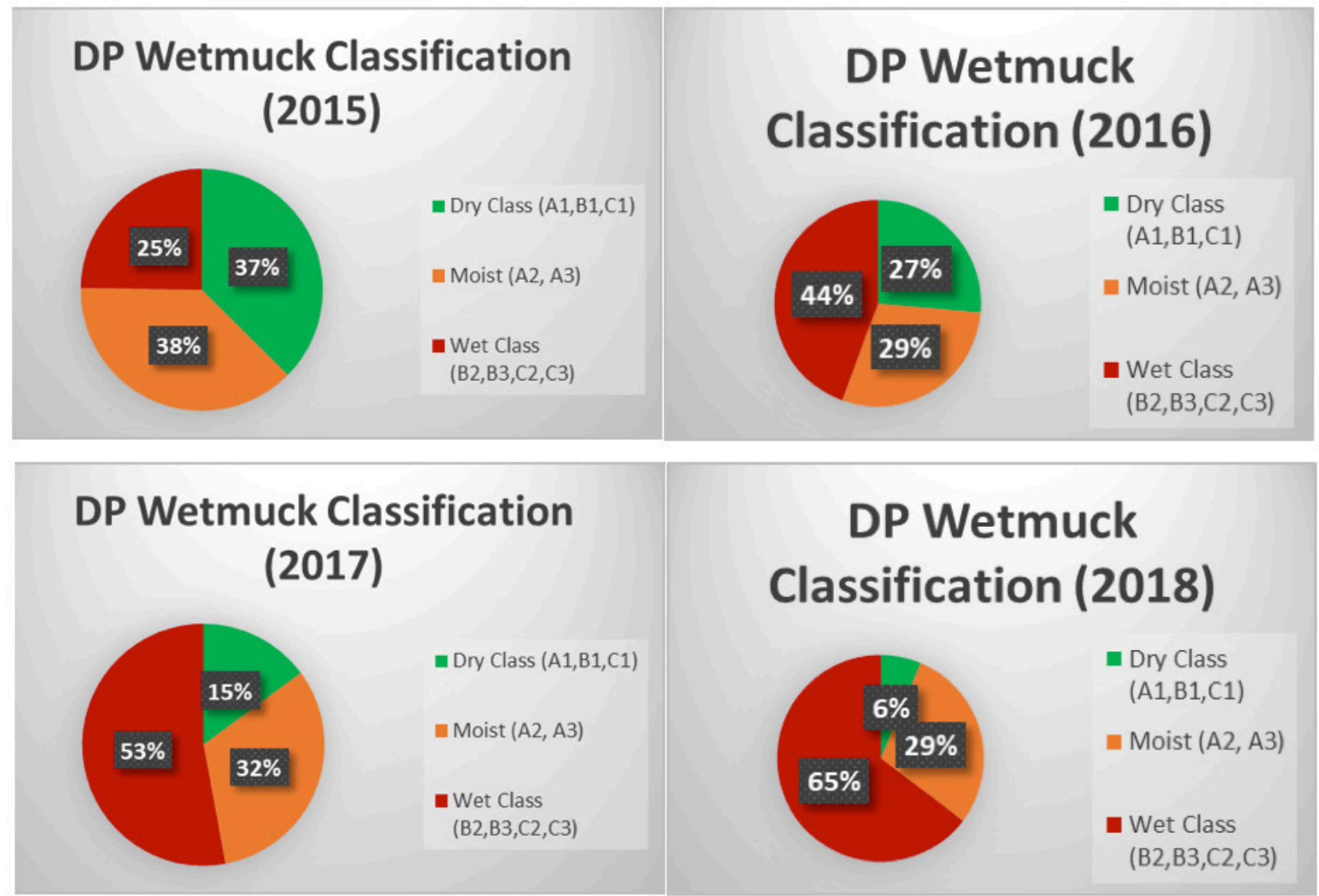

Figure 2 Progression of wet muck drawpoints post mine development

With the increasing number of wet muck drawpoints, the overall tonnage began to decrease and the mine reacted with various improvements to increase the ability to mine the wet ore. Examples of the changes made include the revision of remote barrier placements to enable clean-up of spills faster, improvements in the reliability of the remote loaders, enhancements to the chutes to better contain the wet material and tighter control of the blending to reduce oreflow disruptions. This resulted in a slowing of the declining productivity and a marked increase in the quantity of spills experienced as illustrated in Figure 3 for the 2015 to 2019 period. 


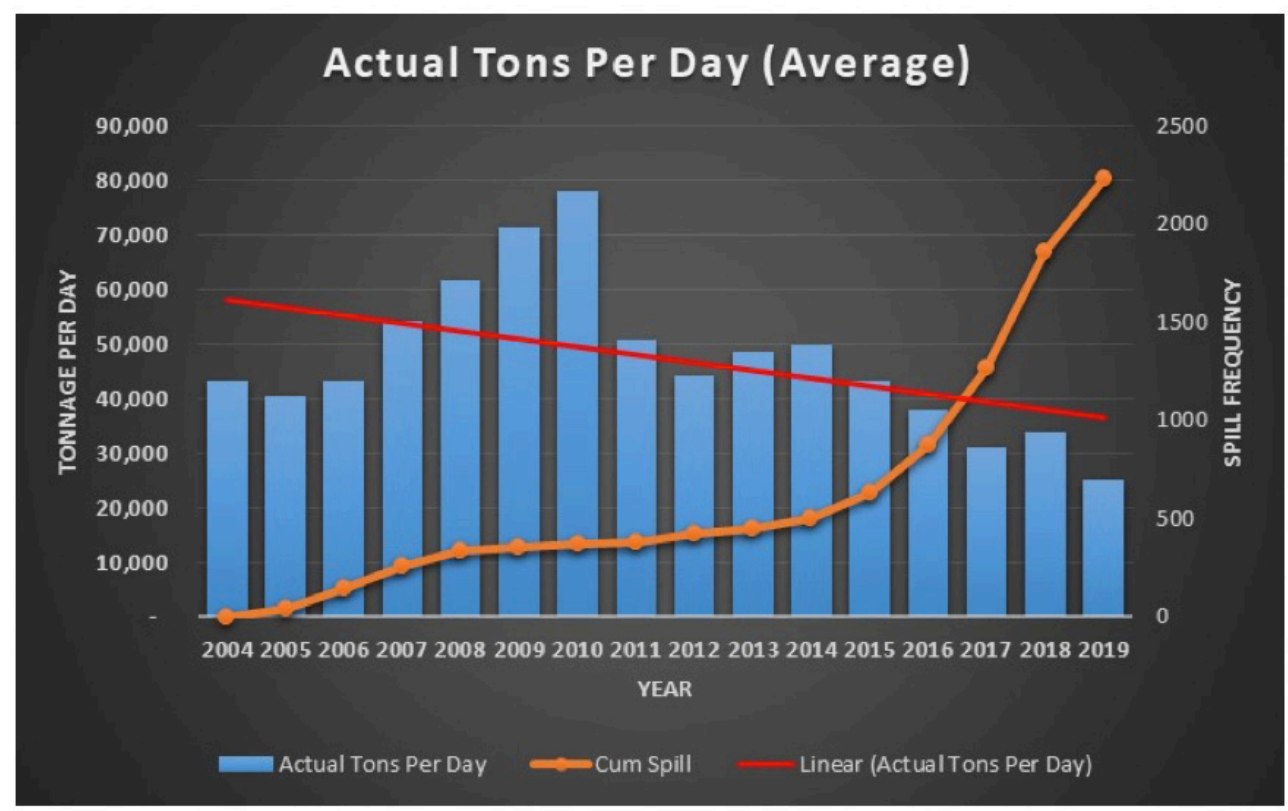

Figure 3 Productivity in comparison to spill frequency

\subsection{Drawpoint large spill risk classification}

In 2018, in addition to the increased spill frequency, DOZ began to experience rare and very large spills, which flowed beyond the wet muck barriers located at the end of the panels. This necessitated an update to the spill classification system utilising historical data on spills in the DOZ to further subdivide wet muck drawpoints based on the likelihood the drawpoint could generate a large spill (Prasetyo 2018). In table 2 below, the example is a C3 drawpoint, that has three closed neighbouring drawpoints, a $300 \mathrm{~m}$ height of draw and has incurred 25 spills to date, the largest of which was estimated at $800 \mathrm{~m}^{3}$ and flowed 110 $\mathrm{m}$. This resulted in a high-risk classification in table 3.

Table 2 Drawpoint hazard classification scheme

\begin{tabular}{|c|c|c|c|c|c|}
\hline Contributing factor & Lower & Middle & Upper & $\begin{array}{c}\text { Weighting } \\
\text { factor }\end{array}$ & Example score \\
\hline DP Class & $\mathrm{A} 1, \mathrm{~A} 2, \mathrm{~B} 1, \mathrm{C} 1$ & $\mathrm{~A} 3$ & $\mathrm{~B} 2, \mathrm{~B} 3, \mathrm{C} 2, \mathrm{C} 3$ & $30 \%$ & $3 \times 0.3=0.9$ \\
\hline Isolated Draw & $0-1 \mathrm{DP}$ & $2-5 \mathrm{DP}$ & $6-9 \mathrm{DP}$ & $20 \%$ & $2 \times 0.2=0.4$ \\
\hline Height of Draw & $0-100 \mathrm{~m}$ & $100-200 \mathrm{~m}$ & $>200 \mathrm{~m}$ & $10 \%$ & $3 \times 0.1=0.3$ \\
\hline Spill Frequency & $<10$ & $10-20$ & $>20$ & $20 \%$ & $3 \times 0.2=0.6$ \\
\hline Spill Volume & $<500 \mathrm{~m}^{3}$ & $\begin{array}{c}500-1,000 \\
\mathrm{~m}^{3}\end{array}$ & $>1,000 \mathrm{~m}^{3}$ & $10 \%$ & $2 \times 0.1=0.2$ \\
\hline Spill Distance & $<75 \mathrm{~m}$ & $75-100 \mathrm{~m}$ & $>150 \mathrm{~m}$ & $10 \%$ & $3 \times 0.1=0.3$ \\
\hline Parameter Score & 1 & 2 & 3 & - & 2.7 \\
\hline
\end{tabular}




\section{Table 3 Risk Classification}

\begin{tabular}{|c|c|c|}
\hline Color & Category & Risk Score \\
\hline & Low Risk & $0-1.5$ \\
\hline & Medium Risk & $1.6-2.0$ \\
\hline & High Risk & $2.1-3.0$ \\
\hline
\end{tabular}

\section{$3 \quad$ Wet muck hazard management}

PTFI has established a Safe Operating Procedure (SOP) for mining wet muck, which seeks to prevent people from working in an area with a spill risk. This is achieved through a combination of remote equipment and time-based exclusions.

\subsection{Secured drawpoints}

Drawpoints within wet muck areas that are planned to be closed temporarily or permanently must be secured. Permanently closed drawpoints are sealed with a concrete wall engineered to withstand anticipated long term loads. Drawpoints closed temporarily are secured by placing $100 \mathrm{~mm}$ of fibrecrete on the drawpoint muck pile. The shotcreted face is also equipped with simple drains, typically plastic pipes inserted into the pile before spraying. The idea being that the drawpoint is metastable and as long as momentum is avoided, the force required to be contained is relatively small. Shotcrete secured drawpoints are formally inspected weekly when work is occurring in a panel. An example is shown in Figure 4.

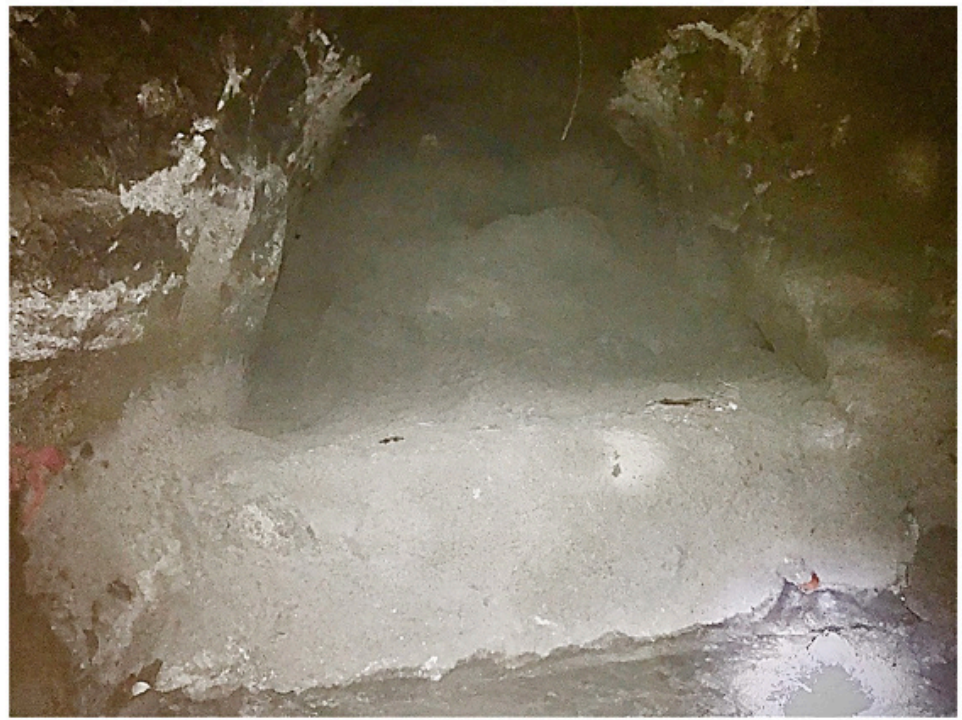

Figure 4 Temporary closed drawpoint, drain pipe visible lower right

\subsection{Time based exclusion}

After each spill, a standardized report is completed and submitted to the geotechnical team that maintains a database of all spills. The report includes the time of the spill and when the drawpoint was last mucked. That allows a review of the lag time between mucking a drawpoint and a triggered spill. The data shows that the vast majority of triggered spills occur within the first two hours, and all triggered spills occur within 24 hours. Although the highest likelihood is that a spill will be triggered in the drawpoint actively mucked, each drawpoint is considered to have an influence area of 9 drawpoints in which a spill could be triggered indirectly. 
All wet muck mining areas, therefore, have a 24-hour exclusion from the last bucket remotely mucked until a trained person may enter the remote mining area to evaluate the condition of the drawpoints and determine if it is safe to work in the panel.

\subsection{Distance exclusion}

Until the massive spills commenced in 2018, all wet muck exclusion was achieved by placing a physical gate at each end of a mining panel that contained active wet muck drawpoints. A flashing strobe provides awareness, and a sign provides information on the reason for the gate. The massive spills however, flowed beyond the exclusion gate and into the panel fringe drives. The longest of these spills had carried substantial flow $150 \mathrm{~m}$ and as a result, drawpoints classified under the large spill hazard assessment as moderate or high-risk require $150 \mathrm{~m}$ stand-off distance. Given that persons must enter within the barrier to set-up remote equipment, the gate must be $170 \mathrm{~m}$ from a medium or high-risk drawpoint before it can be mined. There is no specified distance requirement for low-risk wet muck drawpoints.

\subsection{Haulage level}

Existing chute designs have proven incapable of successfully storing wet muck, loading a truck and then closing again. Although approximately 150 tonnes of wet muck can be held by the existing chutes, if the door is opened to release a 50 tonne truckload it is often not possible to stop the flow and retain the remaining 100 tonnes. The mine therefore operates a 50-tonne in, 50-tonne out process on the wettest material to ensure there is no risk of engulfing a truck. The CAT MineStar system, a commercially available mobile asset monitoring and management system, used for tracking production LHD's at PTFI has been extended to the truck haulage level providing improved management of orepass inventory. Further, trucks no longer drive through the chutes, but back into them so that any spill is away from the cab not toward it. Meanwhile, work continues on the development of a chute capable of both storing and controlling wet muck.

\subsection{Remote equipment}

The extraction of ore from a caving mine requires ore be removed from a drawpoint, and that occasional blockages within the drawpoint be cleared to allow the continued extraction of ore. That ore is then delivered to an orehandling system, and transported to a crushing system before being conveyed to the mill stockpile. In an ideal situation, when wet muck is present, this process could be conducted remotely to ensure no one is within the active panels or wet muck oreflow areas.

Currently, in the DOZ all production LHDs are operated remotely using the MineStar Command System from Caterpillar, and no manual loading occurs. The mine has also developed the ability to handle drawpoint blockages remotely using Getman water cannons equipped with a remote operating system from Remote Control Technologies (RCT) and mobile rock breakers. However, blasting continues to be required, and this is conducted manually after the 24 hour exclusion period.

Remote equipment is used to mitigate the risk to people from exposure to spills, but it does nothing to reduce the risk of spills and consequently remote equipment itself is frequently entrapped.

Recovery of the entrapped equipment is done using remote loaders, as far as practicable, but still occasionally requires people in the panel for excavation and towing. This excavation is completed by a small excavator and in some cases in part by hand. Recovery is through towing with another loader or with a Beltor loader extraction device.

Drawpoint securing is currently completed manually however, the team has worked with Jaycon on the development of a remote shotcreting unit and expects to begin a trial of this process in late 2020. If successful, this will allow drawpoints to be secured before anyone enters a panel. 


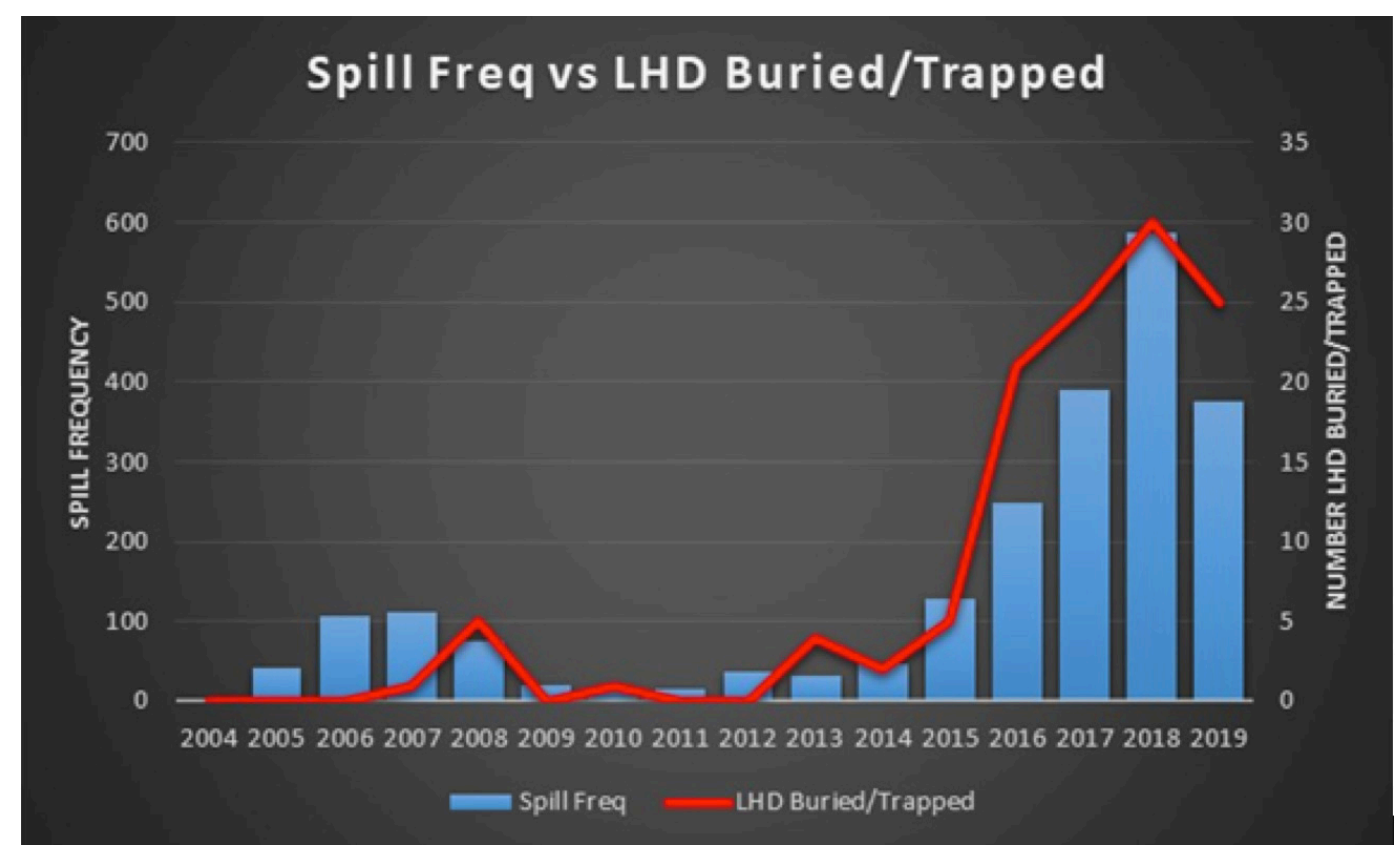

Figure 5 LHD entrapment from spills through Aug. 19

Within downstream oreflow systems, spill recovery is largely completed manually by hand as excavations were not designed originally to accommodate remote excavating or loading equipment in proximity to the oreflow systems.

\section{$4 \quad$ Implications for the future}

The goal is that the future underground caving mines at PTFI will deliver the right tonnes on a continuous basis at the lowest practicable cost.

To achieve this, mines will be operated from a central location on a continuous basis using a combination of autonomous, semi-autonomous and manual equipment controlled through a semi-intelligent production management system.

To this end, wet muck mining has led PTFI to develop the capability of operating LHD's remotely and it now operates the largest fleet of remote underground LHD's in the world. Further, the resulting production interruptions from wet muck mining have guided PTFI on the requirements of a fully remote mining system.

\subsection{Phases of technology application}

The delivery of this goal will be achieved using a series of implementations, some of which are already complete. The first is to be able to track all tonnes from origin to destination to ensure the right tonnes are moved. This project is complete, however, in 2020 a replacement technology was implemented as the existing solution has been obsoleted. The replacement technology substantially lowers the price point to achieve this task.

The second step is to operate remotely to achieve continuous operations and to remove operators from harm's way. For LHD's, this has been achieved with current work focused on updates to improve remote productivity. Ultimately, it is expected that higher productivity will be possible in remotely operated equipment than manually operated machines, as has been achieved in open pits.

The third step is to operate without interruption. A large part of the standby and delay time is caused by implementation of remote capability on only one part of the production cycle (LHD's), necessitating 24- 
hour safety stand downs between mucking and any other activity. This is being addressed by expanding remote capability across the full production cycle.

Once this step is complete, the ability to move the right tonnes is substantially improved since the addition of location and cycle tracking for secondary breaking, recovery and road maintenance equipment and the monitoring of the cave health allows for the optimization of resource assignment for the overall production process as a whole rather than local optimisations, which may be suboptimal for the overall production. It also will establish valuable data sets.

In the two-year horizon, it is expected that loaders will be able to operate the majority of cycles autonomously and in the five-year horizon trucks will be able to complete the majority of their cycles autonomously as well. This provides continuous operation with minimal harm to the equipment.

Finally, as both data sets and equipment capability expand, the amount of decisions and consequent actions that can be optimized autonomously and continuously will become substantial.

\subsection{Remote operating room}

The remote operating room (ROR) is the single location from which the $D O Z, G B C$ and $D M L Z$ are operated as a predictable and reliable underground mining operation. The intent is that the full production cycle from cave to crusher is operated in a common area with clear visibility of the equipment and the people within the autonomous mining area. This visibility includes their location, activities and health. In addition, the health of the cave itself is also monitored to ensure the appropriate part of the cycle is being optimized to assure a consistent "manufacturing" of ore.

The ROR will house operator stations, maintenance health stations, mine controller stations and system technicians. The majority of stations are designed to allow for the physical operation of the equipment, using controls that resemble those on the actual machine. Stations for maintenance health, mine controller and system technician are operations centre-type desks with multiple monitors displaying the relevant real-time information for each role and communications equipment. For autonomous machines, operator stations resemble those of the mine controllers and each operator oversees many machines simultaneously. In the medium-term this fully autonomous operation is expected to be achievable but is not part of the initial deployments.

\subsection{Production management system}

The production management system will provide the mine controller a complete picture of the location, state (e.g. standby) and performance of all people and assets within the production areas as well as the general condition of the cave and panels. This information will be presented in general, graphical format enabling interpretation at a glance and better, faster decisions on work assignments. Further, much of the assignment will be handled automatically by the software reducing the burden on the controller to exceptions only. Equipment within the production areas will also have a clear view of their location as well as that of equipment or people within their area and the state of the zones in their vicinity (remote or manual).

\subsection{Area management}

The extraction level will include autonomous, remote and manual work within the same environment. This area will be described broadly as the Autonomous Mining Area (AMA). All entities within the AMA will be equipped with SafeDetect (a Minetec product) and will be managed by the Area Isolation Management System (AIMS). AIMS is being developed in partnership with Caterpillar for trial in late 2020 and allows both dynamic and remote changes in the allocation of sections of the mine to manual, remote or autonomous use. The boundaries of the AMA will be the only locations deploying physical barricades and those barricades demarcate the point beyond which entities must have the appropriate systems onboard and in which all persons entering or operating require competence in AIMS and AMA procedures. 


\subsection{People}

The operation and maintenance of the systems associated with the highly mechanized, $100 \%$ network dependent production system will require a revision to organisation structure and skilled specialists who have attained a defined level of competence. The people who are engaged in developing, deploying, operating and maintaining the mine's technology are what is broadly described as the Technology Group. This group includes Remote Plant, Remote Solutions, Remote Operations and Remote Projects.

Remote Plant includes the configuration and maintenance of the physical componentry in the field. Remote Solutions includes the operation and maintenance of specialized networking, communication systems and their associated hardware as well as the management of the operating applications and massive volumes of data generated by their operation. Remote Projects is accountable to work in collaboration with OEM's establishing the development, project execution and operational readiness plans. The group also secures funding and project manages the initial deployment, as well as ensuring operations are sufficiently staffed, trained and provided with the appropriate systems for continuous operation of the completed projects. Remote Operations is accountable for the use of the remote equipment and systems to maximize safe production at the lowest possible cost. The team is a virtual team with members in multiple sub sections of the business.

\section{Conclusions}

The mining of wet muck is a challenging undertaking with substantial safety hazards that must be managed. PTFI has developed sufficient technical ability and empirical knowledge to safely operate a wet muck mine. The optimisation of this mine and further reduction of risk both point to the need to develop a continuous and largely remote mine with substantial autonomous capability. PTFI is well on track to achieving the first generation of this mine within the five year time horizon and is already operating the largest remote underground fleet in the world.

\section{Acknowledgement}

The authors would like to thank PTFI management for allowing the publication of this paper; the many people involved in mining wet muck safely on a daily basis and whose observations have helped develop the systems in place and the OEM's for collaborating with PTFI on this journey towards continuous remote mining.

\section{References}

Call \& Nicholas Inc. 1998, 'IOZ Wet Muck Study'.

Casten, T, Rachmad, L, Arkadius, T, Osborne, K \& Johnson, M 2008, 'P.T. Freeport Indonesia's Deep Ore Zone Mine - expanding to 80,000 tonnes per day'.

Learning and Organisational Development 2019, 'Wet Muck Awareness Training', Power Point Presentation.

Prasetyo, R 2018, 'Massive Spill in 1A \& Resulting Actions', Power Point Presentation. 